\section{e.l.f. Hiss modulation at harmonics of the helium gyrofrequency}

MANY plasma-wave phenomena involving the proton gyrofrequency have been observed in the ionosphere and magnetosphere but very few involving the helium gyrofrequency. One such is the phenomenon known as helium whistlers which were discovered by Barrington, Belrose and Mather ${ }^{1}$ with the Canadian Alouette II satellite. Horita and Friesen ${ }^{2}$ have also reported fine structure at harmonics of the proton and helium gyrofrequency on whistlers detected by the v.l.f. receiver on board the Canadian Isis II satellite. Another paper ${ }^{3}$, reporting proton gyrofrequency phenomena on auroral hiss, mentioned parenthetically that extra low frequency hiss modulated at harmonics of the helium gyrofrequency has been observed. Here I give further details of the e.l.f. hiss modulation and discuss the region where the modulation may possibly have occurred.

Figure 1 shows the v.l.f. spectrogram made at the Quito station from the Canadian Isis II satellite on April 6, 1971 at 2329.00 to $2329.15 \mathrm{UT}$; at 2329.10 UT the satellite was at $8.5^{\circ} \mathrm{S}$, $74.1^{\circ} \mathrm{W}$, and at an altitude of $1,379 \mathrm{~km}$. The superimposed horizontal lines indicate the harmonics of the helium gyrofrequency $f_{\mathrm{He}}=112.5 \mathrm{~Hz}$ (which corresponds to an observed proton gyrofrequency $f_{\mathfrak{p}}=4 f_{\mathrm{He}}=450 \mathrm{~Hz}$ ). This value was found by examining the spacing of the e.l.f. hiss modulation and is greater than the value of $60.7 \mathrm{~Hz}$ for the local helium gyrofrequency in the immediate vicinity of the satellite, suggesting that the source region is at a location or locations beneath the satellite. Note that the horizontal lines generally lie in the gaps of the e.l.f. hiss modulation for frequencies $\gtrsim 2 f_{\mathrm{p}}$ and lie on the signals for frequencies $<2 f_{\mathrm{p}}$. The lower frequency boundary of the e.l.f. hiss seems to be close to the local proton gyrofrequency of $243 \mathrm{~Hz}$.

Also shown are whistlers exhibiting the fine structure related to the helium gyrofrequency reported earlier ${ }^{2,3}$. Note that the whistler to the left at about 2329.05 ur has fine structure such that the horizontal lines pass through the gaps, indicating interaction at a location where the helium gyrofrequency is $112.5 \mathrm{~Hz}$, the same as for the e.l.f. hiss modulation, suggesting a relationship between the two phenomena. The same feature is seen on the whistler to the right at about 2329.10 UT for frequencies $>2 \mathrm{kHz}$. Below $2 \mathrm{kHz}$ the signal, or perhaps another signal, seems to be displaced a fraction of a second to

Fig. 1 e.l.f. hiss exhibiting modulation related to the helium gyrofrequency. The fine structure leads to a helium gyrofrequency $f_{\mathrm{He}}=0.25 f_{\mathrm{p}}=112.5 \mathrm{~Hz}$. which is greater than the local value of $60.7 \mathrm{~Hz}$ in the immediate vicinity of the satellite; the superimposed horizontal lines are at harmonics of $f_{\mathrm{He}}$. Also shown are whistlers which may be related to the e.l.f. hiss.

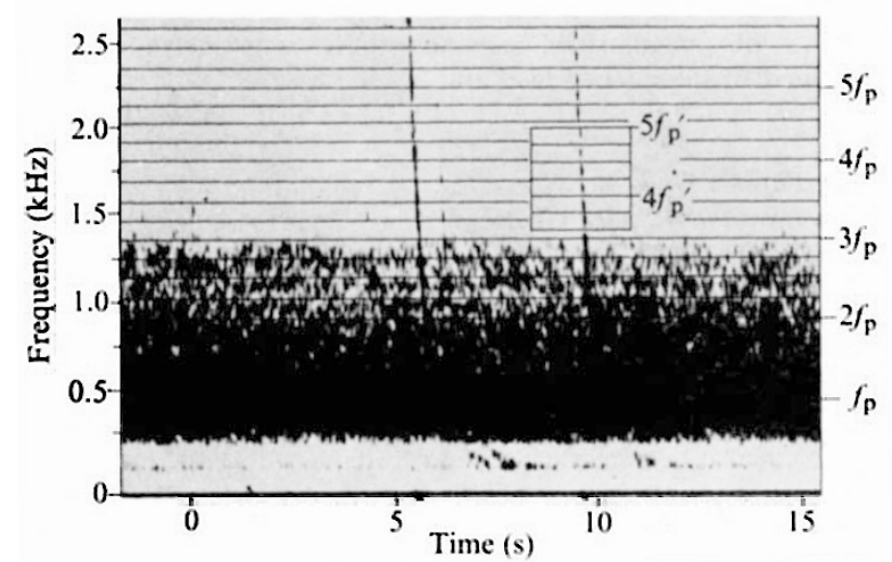

the right and has fine structure leading to a helium gyrofrequency of $\sim 100 \mathrm{~Hz}$ corresponding to $f_{\mathfrak{p}}{ }^{\prime}=400 \mathrm{~Hz}$, suggesting a slightly higher interaction region than that for the other whistler. Horizontal lines at these harmonics pass through the gaps in the whistler, as before. This feature suggests attenuation of the whistler at the harmonics of the helium gyrofrequency. For the very faint whistler at about 2329.06 UT, however, the lines pass through the signals, suggesting enhancement at integral values of $f_{\mathrm{He}}$. To be cautious, however, although the fine-structure frequency has been carefully scaled to $\lesssim 1.5 \%$ accuracy here, a difference of $2.5 \%$ would shift the 20 th helium gyrofrequency harmonic line $\left(5 f^{\prime}\right.$ p) by half a harmonic so that the gaps of the whistler in the box would lie between rather than on the harmonic lines. The phenomenon must, nonetheless, be related to the helium gyrofrequency, and for those examples with a large number of harmonics the very high order harmonic frequencies can be more accurately determined.

Other examples of the e.l.f. hiss modulation gave values for the observed helium gyrofrequency which were approximately equal to and also less than the local value in the immediate vicinity of the satellite, depending on whether the satellite was somewhat near the plasmapause or outside the plasmasphere respectively. These observations are consistent with the statement by Taylor and Shawhan ${ }^{4}$ that the propagation of e.l.f. hiss is downgoing outside the plasmapause and upgoing inside.

Since the ell.f. hiss modulation provides a signature for the source region or regions where the modulation took place, it provides a means for studying the possible generation or interaction region of the e.l.f. hiss depending on whether the modulation took place at the same time as or after the ell.f. generation. A preliminary calculation for the altitudes of the source regions, assuming wave propagation along the magnetic field of the Earth, gave $\sim 2,300 \mathrm{~km}$ outside the plasmasphere, $500 \mathrm{~km}$ inside the plasmasphere and $1,400 \mathrm{~km}$ (the Isis II satellite altitude) near the plasmapause. Obtaining the observed gyrofrequency used to calculate the location of the generation or interaction region can be very complex since at times three or more components may be seen on the v.l.f. spectrograms, indicating a superposition of many e.l.f. waves from a number of source locations.

Work is in progress to analyse more examples of e.l.f. hiss modulation. The results of the analyses and more complete details of the items mentioned above will be published elsewhere. Further studies of the phenomenon, especially with data from satellites at other altitudes than the nearly constant Isis II satellite altitude of $1,400 \mathrm{~km}$, may provide further clues as to the generation mechanism for e.l.f. hiss, the subject of some discussion in very recent papers ${ }^{5-9}$.

I thank Mr Lane Friesen for assistance in the preparation of Fig. 1, Drs R. E. Barrington and F. H. Palmer for the microfilmed Isis II v.l.f. spectrograms, the World Data Center A for Rockets and Satellites at the Goddard Space Flight Center for supplying microfilmed world maps for the Isis II satellite and the NRC of Canada and the University of Victoria for support.

\section{R. E. HORITA}

Department of Physics,

University of Victoria,

Victoria, British Columbia, Canada

Received March 15; accepted April 15, 1976.

1 Barrington, R. E., Belrose, J. S., and Mather, W. E., Nature, 210, 80-81 (1966).

2 Horita, R. E., and Friesen, Lane, J. atmos. terr. Phys., 37, $1497-1500$ (1975).

3 Horita, R. E., Friesen, Lane, and Chan, A. W. Y., J. atmos. terr. Phys. (in the pressi.

Taylor, W. W. L., and Shawhan, S. D., J. geophys. Res., 79, 105-117 (1974)

5 Russeli, C. T., McPherron, R. L., and Coleman, P. J., Jr, Space Sci. Rev., 12, 810-856 (1972)

Thorne, R. M., Smith, E. J., Burton, R. K., and Holzer, R. E., J. geophys. Res., $78,1581-1596(1973)$

Etcheto, J., Gendrin, R., Solomon, J., and Roux, A., J. geophys. Res., 78, 81508166 (1973).

8 parady, B. K., et al., J. geophys. Res., 80, 2183-2198 (1975).

9 Thorne, R. M., and Barfield, J. N., Geophys. Res. Lett., 3, 29-32 (1976). 